Rodríguez, M. S., Buitrago, A., Varón, N., y Quintero, R. (2019). La satisfacción de los usuarios afiliados al sistema de salud en la ciudad de Ibagué, Colombia. Revista Lebret 11, 123 - 147. https://doi.org/10.15332/rl.v0i11.2415

Artículo de investigación:

\title{
La satisfacción de los usuarios afiliados al sistema de salud en la ciudad de Ibagué, Colombia
}

\section{The satisfaction of users affiliated with the health system in the Ibagué city, Colombia}

\author{
Mario Samuel Rodríguez Barrero \\ Aracelly Buitrago Mejía \\ Nubia Varón Triana \\ Ramiro Quintero García
}

\begin{abstract}
Resumen
Este artículo presenta un análisis sobre la percepción de los usuarios de los servicios de salud en Ibagué, Colombia. El marco teórico se basa en la teoría de sistemas y postulados sobre calidad y servicio. El estudio es descriptivo con enfoque cuantitativo. La población está constituida por los afiliados al sistema de salud en la ciudad y la muestra corresponde a 384 usuarios. El nivel de confianza para la muestra es del 95\% y el margen de error es del 5\%. La información primaria se obtiene a través de una encuesta que contiene 5 variables: comunicación, oportunidad, profesionalismo, infraestructura y eficiencia. Los resultados muestran que el $44.5 \%$ de los usuarios están satisfechos con los servicios de salud y el $29 \%$ no están satisfechos. Finalmente, se concluye que las principales fallas que los usuarios perciben están en comunicación, infraestructura, humanización y tiempo de espera.
\end{abstract}

\section{Palabras clave}

Calidad de la atención médica, gestión de servicios de salud, humanización del servicio de salud, satisfacción de los usuarios, sistema de salud colombiano.

\section{Códigos de clasificación JEL: I19, I3, H75}

\begin{abstract}
This paper presents an analysis on the perception of health service users in Ibague, Colombia. The theoretical framework is built based on the theory of systems and postulates about quality and service. The study is descriptive with quantitative approach. The population is constituted up of all members of the health system in the city and the sample corresponds to 384 users. The level of confidence for the sample is $95 \%$ and the margin of error is $5 \%$. The primary information is obtained through a survey that contains 5 variables: communication, opportunity, professionalism, infrastructure and efficiency. The results show that $44.5 \%$ of users are satisfied with health services and $29 \%$ are dissatisfied. Finally, it is concludes that the main failures that users perceive are in communication, infrastructure, humanization and waiting time.
\end{abstract}

\section{Keywords}

Quality of health care, health services management, humanization of health service, users' satisfaction, health system colombian.

1 Universidad Cooperativa de Colombia. Correo electrónico: mario.rodriguezb@campusucc.edu.co Código ORCID: 0000-0001-9356-6764.

2 Universidad del Tolima. Correo electrónico: abuitragom@ut.edu.co

Código ORCID: 0000-0002-6583-7827.

3 Universidad Cooperativa de Colombia. Correo electrónico: nubia.varont@campusucc.edu.co Código ORCID: 0000-0001-5069-1821.

$4 \quad$ Universidad del Tolima; Correo electrónico: rquinter@ut.edu.co 


\section{Introducción}

En este artículo se presentan los resultados del "Estudio técnico del sector salud en la ciudad de Ibagué", investigación auspiciada por la Universidad Cooperativa de Colombia y la Universidad del Tolima. La importancia del tema se deriva del papel del sector salud en los aspectos sociales y económicos del país, así como de la crisis que atraviesa, lo cual afecta la prestación de los servicios de salud y, en consecuencia, la satisfacción de los usuarios, aspecto que reviste gran sensibilidad por tratarse de la salud humana (Álvarez, García y Londoño, 2016). Además, los problemas sociales caracterizados por las profundas desigualdades, desempleo y pobreza en Colombia, hacen de este un tema de interés nacional (Yepes y Marín, 2018).

Inicialmente, se presenta una argumentación desde la perspectiva legal y social en el ámbito global y nacional, abordando las principales problemáticas del tema objeto de estudio, con lo cual se soporta en lo teórico, que se fundamenta en los postulados de la gerencia del servicio, la medición de satisfacción, los lineamientos de la auditoría en salud y la humanización de los servicios, elementos que dan lugar a la operacionalización de las variables y exposición de los aspectos metodológicos que, a su vez, preceden la presentación, análisis y discusión de los resultados.

Desde la perspectiva global, la calidad en los servicios de salud es considerada como una prioridad, según se refleja en la Declaración de los Objetivos de Desarrollo Sostenible - ODS, en la cual se considera imprescindible mantener la calidad en los servicios de salud para garantizar una vida saludable y promover el bienestar de los ciudadanos. Este propósito se materializa en la Meta 3.8 de los ODS, que consiste en "lograr la cobertura sanitaria universal, en particular la protección contra los riesgos financieros, el acceso a servicios de salud esenciales de calidad y el acceso a medicamentos y vacunas seguros, eficaces, asequibles y de calidad para todos" (Organización de Naciones Unidas - ONU, 2015). En esta declaración, se evidencia la importancia de la calidad en los servicios de salud, que constituye un aspecto determinante en la satisfacción de los usuarios, la cual depende de las experiencias que ellos tienen al hacer uso de estos servicios.

Según Syed, Leatherman, Mensah-Abrampah, Neilson y Kelley (2018), la Organización Mundial de la Salud - OMS, el Banco Mundial y la Organización para la Cooperación y el Desarrollo Económico - OCDE consideran indispensable garantizar la prestación de servicios de salud con calidad y lograr la cobertura universal, además, desde estas instituciones se ha manifestado la preocupación respecto al logro de los ODS en lo referente a las problemáticas en salud, por lo que han solicitado que la calidad sea considerada como elemento fundamental para la prestación y medición de estos servicios.

En Colombia, desde la Constitución de 1991 (artículo 49), la salud era considerada como un servicio, pero a partir de la Ley 1751 de 2015, pasa a ser reconocida como un derecho fundamental que debe ser protegido, regulado y garantizado por el 
Estado, motivando con este reconocimiento al Gobierno y las empresas prestadoras, a facilitar el acceso a los servicios de salud, fortaleciendo el control de precios y la incorporación de nuevas tecnologías, así como la evaluación del servicio en términos de disponibilidad, aceptabilidad, accesibilidad y calidad, aunque en la actualidad, el Estado dista de ser garante de este derecho, como lo reconoce Pretelt (2017).

Hace más de una década en Colombia se reconoce la necesidad de tomar medidas para prestar atención efectiva, segura, eficiente, accesible, apropiada y satisfactoria a los usuarios del sistema de salud colombiano, así como la urgencia de incorporar mecanismos para promover la garantía de la calidad, entendida como una función esencial de la salud pública (Kerguelén, 2008), sin embargo, esta necesidad no se ha materializado en acciones concretas que mejoren sustancialmente la atención en los servicios según los estudios recientes (Morales y García, 2017; Suárez, Puerto, Rodríguez y Ramírez, 2017; Triviño, 2015).

Para el 2016, el Ministerio de Salud y de la Protección Social promulgó la Política de Atención Integral en Salud - PAIS y el Modelo Integral de Atención en Salud MIAS, mediante la Resolución 429 de 2016, para organizar un sistema que, de manera coordinada y armónica, materializara el derecho fundamental a la salud y se mejoraran las condiciones de prestación del servicio, sin embargo, estas acciones no han sido lo suficientemente efectivas, pues aún son constantes las problemáticas e inconvenientes de usuarios que manifiestan su inconformismo, los que no se solucionan solo con una nueva ley, sino con una reforma estructural, según lo concluye Moreno (2016).

La importancia del Sistema de salud subyace en su impacto económico y social, razón por la que el 5.9\% del gasto corriente del PIB corresponde a este rubro según un informe de la Organización Mundial de la Salud - OMS y la Organización Panamericana de la Salud - OPS (2018). Además, este sector provee más de 667 mil empleos en el país (Ministerio de Salud, 2017) y cuenta con más de 47 millones de afiliados (Ministerio de Salud, 2019), según se observa en la tabla 1.

Tabla 1. Afiliados al Sistema de Seguridad Social en Salud

\begin{tabular}{cccc}
\hline Tipo de afiliación & Diciembre 2017 & Diciembre 2018 & Abril 2019 \\
\hline Sisbén 1 & 25.871 .020 & 27.140 .038 & 27.140 .038 \\
\hline Sisbén 2 & 2.811 .274 & 2.787 .974 & 2.787 .974 \\
\hline Población pobre no afiliada & 554.364 & 525.698 & 312.696 \\
\hline Contributivo & 22.045 .454 & 22.378 .384 & 22.424 .262 \\
\hline Subsidiado & 22.434 .577 & 22.658 .108 & 22.814 .565 \\
\hline Excepción y especiales & 2.287 .296 & 2.138 .117 & 2.144 .376 \\
\hline Afiliados & 46.767 .327 & 47.174 .609 & 47.383 .203 \\
\hline Total población & 49.291 .609 & 49.834 .240 & 50.013 .241 \\
\hline Cobertura & $94,88 \%$ & $94,66 \%$ & $94,74 \%$ \\
\hline
\end{tabular}

Fuente: Ministerio de Salud (2019). 
Con relación a las problemáticas en salud, la OMS y la OPS (2018) indican que por cada cien mil nacidos vivos, mueren 54 madres y cerca de 355 fallecen anualmente por causas relacionadas con el embarazo y el parto; en el citado informe también se indica que 17 niños fallecen por cada mil nacimientos y 19 mueren por cada mil antes de llegar a los cinco años, tasa muy alta comparada con países como Ecuador $(11,5)$ y Puerto Rico (9).

En cuanto a cobertura, el indicador para Colombia es positivo con un 92\%, según la OMS y OPS (2018), sin embargo, un estudio realizado por Ayala (2014), con cifras del Departamento Administrativo Nacional de Estadística - DANE recolectadas entre 1997 y 2012, revela que el acceso a los servicios médicos en Colombia se redujo de $79.1 \%$ a $75.5 \%$ en este período, lo que corresponde a las personas que efectivamente podían acceder a los servicios médicos cuando lo requerían, lo cual permite colegir que, además de la cobertura, se deben eliminar otras barreras que dificultan el acceso a los servicios, sea por costos, ubicación o congestión en el sistema.

En lo referente a las dificultades del Sistema se encontró que, en 2019, el número de quejas, peticiones y denuncias ante la Superintendencia fue de 694.068, de las cuales el $83 \%$ hacían referencia a las restricciones que perciben los usuarios para acceder a los servicios y un $12 \%$, a la insatisfacción con el proceso administrativo y falta de efectividad en la prestación del servicio, según se muestra en la tabla 2.

Tabla 2. Peticiones, quejas y denuncias por macromotivo

\begin{tabular}{ccc}
\hline Macromotivos & Total 2019 & $\begin{array}{c}\text { Porcentaje de } \\
\text { participación }\end{array}$ \\
\hline Restricción en el acceso a los servicios de salud & 576.374 & $83,0 \%$ \\
\hline Insatisfacción del usuario con el proceso administrativo & 54.836 & $7,9 \%$ \\
\hline Deficiencia en la efectividad de la atención en salud & 28.267 & $3,1 \%$ \\
\hline No reconocimiento de las prestaciones económicas & 27.069 & $0,7 \%$ \\
\hline Peticiones y reclamos interpuestos por IPS-EPS, entidades \\
territoriales y organismos de control y vigilancia
\end{tabular}

Fuente: Superintendencia de Salud (2019).

Según Prada y Salinas (2016), el Comité Editorial de la Revista Gerencia y Politicas de Salud (2015) y el estudio realizado por los autores, entre las principales fallas del SGSS se encuentran las siguientes:

- Ausencia de estrategias efectivas para generar competencia entre EPS

- Insolvencia financiera debido a las dificultades en la gestión de pagos 
- Fallas en el control de gastos e inversiones de las EPS

- Baja calidad en la prestación de los servicios

- Dificultades, congestión y restricciones que dificultan acceder al servicio

- Falta de unidad de criterio en políticas del Plan Obligatorio de Salud - POS

- Ausencia de planes de contingencia para atender períodos de alta demanda

- Fallas en los flujos de información dentro del sistema.

Como consecuencia, los usuarios perciben que se está vulnerando su derecho fundamental a la salud y acuden a la tutela como mecanismo para reclamar sus derechos ante la justicia, congestionando el sistema judicial, pues según el Informe de la Defensoría del Pueblo (2019), el número de tutelas en salud para el 2018 fue de 207.734, lo que representa el 5.2\% del total de tutelas en Colombia y un incremento del $5 \%$ respecto al año anterior.

Según este informe, la EPS con mayor número de tutelas fue Medimás-Cafesalud-Saludcoop con 31.468 tutelas que representa el 5.2\%, seguida de Coomeva con el $5.1 \%$ y Nueva EPS con el $4.4 \%$ del total de tutelas del país, en las que se reclamaba el derecho fundamental a la salud en cerca del $80 \%$ de los casos, y el mínimo vital, derecho de petición, derecho a la vida y seguridad social, en los demás casos, resultando las decisiones a favor de los peticionarios en más del $85 \%$, cifra que revela la necesidad de adoptar medidas para evitar este desgaste del aparato judicial y sobrecostos al sistema de salud. Por lo anterior, se presenta la pregunta ¿Cuál es el nivel de satisfacción de los usuarios de los servicios de salud en Colombia? Planteamiento que orienta la presentación, análisis y discusión de los resultados.

\section{Aspectos teóricos}

Una vez acotada la problemática de estudio, se analizan los elementos teóricos que soportan la investigación, iniciando desde la teoría de los sistemas hasta los elementos que definen la calidad en salud y satisfacción de los usuarios. Según la teoría sistémica Bertalanffy (1992), un sistema está constituido por un grupo de elementos que interactúan entre sí y modifican su comportamiento según estas relaciones. Para Ramos (2011) un sistema, además de su interacción dinámica, se caracteriza porque funciona en conjunto, tiene una estructura definida, se relaciona con el medio y tiene un propósito preciso.

Desde esta perspectiva, se considera que el sector salud funciona como un sistema, porque está conformado por el conjunto de entidades que interactúan para prestar un servicio (Gómez, Pelcastre, y Parada, 2016). Además, esta visión permite entender el alcance de las relaciones que surgen entre los actores del SGSSS y su 
interdependencia, no solo en su interior, sino con el entorno, la sociedad, que es beneficiada o afectada por sus resultados (Gayeski, Parizoto, Guimarães, Erdmann y Meirelles, 2012).

En Colombia, el sistema de salud está conformado por seis tipos de instituciones:

- Las aportantes, como el Estado a través del Fosyga, los empleados, empleadores e independientes.

- Las aseguradoras, Empresas Promotoras de Salud (EPS), Administradoras del Régimen Subsidiado (ARS) y particulares.

- Las Instituciones Prestadoras de Servicios de Salud (IPS).

- Los proveedores de medicamentos.

- Los usuarios (pacientes).

- Las instituciones encargadas de la supervisión, inspección, vigilancia y control (Ministerio de la Protección Social, 2004).

En la figura 1. se presenta una representación de la forma en que funciona el sector salud, como un sistema.

Figura 1. Visión del sector salud como un sistema

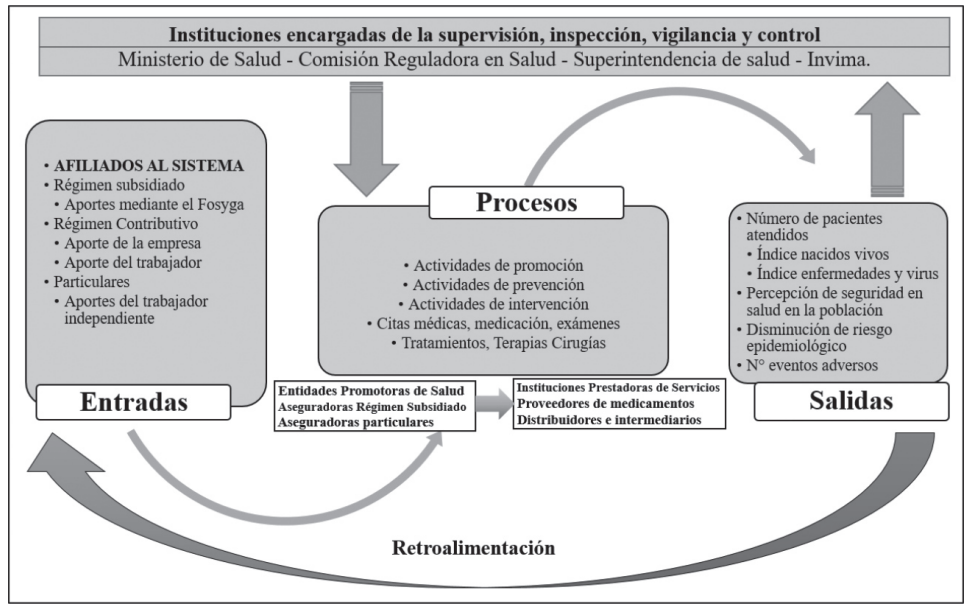

Fuente: Elaboración propia.

Esta visión sistémica del sector salud permite comprender el efecto de las fallas que se presentan en el sistema, y demuestra cómo repercuten en el resto de los actores del sistema y en la percepción negativa de los usuarios, si se presentan fallas en los procesos relacionados con ellos. En este contexto, Donabedian (1986 y 1992) conceptúa que la calidad en salud está determinada por el proceso de atención que 
el paciente requiere para mejorar su estado, teniendo en cuenta todas las partes que intervienen durante este. A partir de este concepto de calidad en salud, Donabedian propone la siguiente clasificación, a partir de la percepción de quien la evalúa:

- Calidad absoluta: determinada por el mejoramiento de la salud del paciente

- Calidad individual: definida por sus expectativas y valoración sobre costos, beneficios y riesgos asociados, a partir de la información recibida.

- Calidad social: se valora en función del beneficio que recibe una población al menor costo social posible; eficiencia en distribución para toda la población.

En efecto, la OMS (1991) conceptúa que la calidad de la atención en salud consiste en "asegurar que cada paciente reciba el conjunto de servicios diagnósticos y terapéuticos más adecuado para conseguir una atención sanitaria óptima", aunado a lo cual, "se deben considerar los diferentes factores que intervienen en el proceso para lograr los mejores resultados, disminuyendo los riegos y buscando la mayor satisfacción del paciente". Igualmente, Palmer (1989) define la atención en salud como la disposición de servicios que sean accesibles y equitativos, ofrecidos con personal profesional excelente, garantizando eficiencia en el uso de los recursos y logrando la satisfacción del usuario.

Por su parte, Donabedian (1996) define tres componentes que permiten medir la calidad de los servicios de salud: estructura, proceso y resultado. Además, en su conferencia "La dimensión internacional de la salud", considera que, para la medición en salud, se deben tres componentes: la atención técnica, el manejo de la relación interpersonal y el entorno, los cuales son permeados por la cultura del país donde se preste el servicio. Teniendo en cuenta estos elementos, se construyó una aproximación al sistema de atención en salud, según se presenta en la figura 2.

Figura 2. Sistema de atención en salud y garantía de calidad

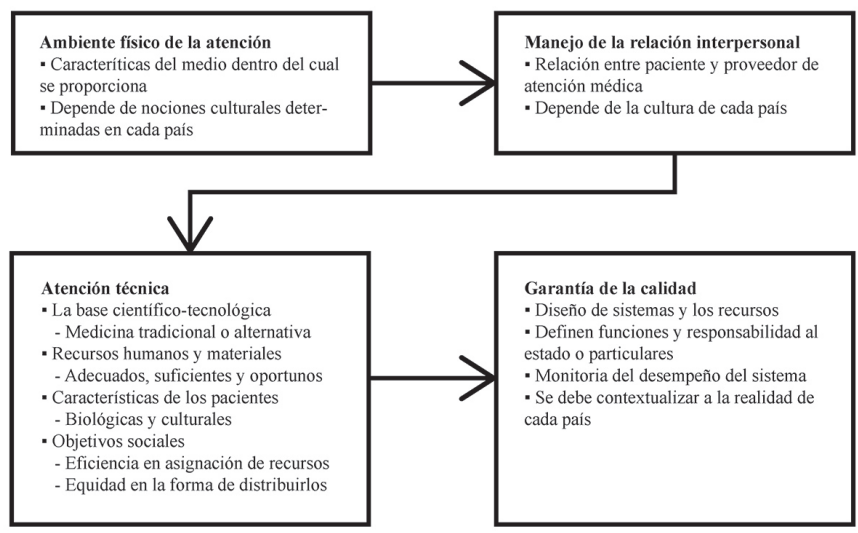

Fuente: Elaboración propia con conceptos de Donabedian (1986, 1992 y 1996). 
Se observa que el concepto de calidad ha tenido una constante evolución, principalmente desde la era industrial hasta la actual y que son diversos los enfoques para definirla, sin embargo, estos coinciden en que su propósito final, es la satisfacción del usuario-paciente, lo cual se logra mediante una atención adecuada, sin embargo, es de anotar, que esta evaluación puede variar dependiendo de la percepción de quien la evalúe, pues está condicionada por diferentes factores y niveles de importancia, lo cual permite colegir, que la percepción de la calidad en salud depende de múltiples atributos y de su valoración, los cuales se modifican según el conocimiento de quien la evalúa, por lo cual se pueden presentar diferentes resultados por parte de quien ofrece y quien recibe el servicio, aunque estén evaluando el mismo (González, Valecillos, y Hernández, 2013).

\section{Metodología}

La investigación se clasifica como descriptiva, por cuanto permite conocer de fuente primaria la percepción de los usuarios respecto a las variables identificadas (Díaz-Narváez y Calzadilla, 2016); la unidad de análisis es el sector salud de Ibagué, Colombia, cuya población corresponde a los 544.475 personas afiliadas al momento de realizar el estudio; de esta población, 347.196 pertenece al régimen contributivo, cifra que corresponde al $63.7 \%$, mientras 184.429 que representan el $34 \%$ hacen parte del régimen subsidiado y 12.850 , cifra que corresponde al $2.3 \%$ restante, pertenece al régimen especial, según se observa en el Informe Cifras de Aseguramiento del Ministerio de Salud y Protección Social (2019).

Para la determinación de la muestra, se consideró un nivel de confianza del 95\%, un error de 5\% y un $\mathrm{Z}$ de 1.96 que corresponde a una distribución de una curva normal; con estos estadísticos se aplicó la fórmula para poblaciones finitas y se obtuvo una muestra poblacional de 384 personas, la cual se distribuyó como se muestra en la tabla 3.

Tabla 3. Distribución de encuestas aplicadas por EPS

\begin{tabular}{ccc}
\hline EPS de afiliación & Proporción & Número de encuestados \\
\hline Medimás & $27 \%$ & 104 \\
\hline Salud Total & $23 \%$ & 88 \\
\hline Nueva EPS & $14 \%$ & 54 \\
\hline Coomeva & $11 \%$ & 42 \\
\hline Sanitas & $9 \%$ & 35 \\
\hline Emcosalud & $7 \%$ & 27 \\
\hline Famisanar & $4 \%$ & 15 \\
\hline Régimen especial & $5 \%$ & 19 \\
\hline Total & $100 \%$ & 384 \\
\hline
\end{tabular}

Fuente: Elaboración propia. 
En cuanto a la técnica para la selección de las unidades muestrales, se seleccionó el tipo de muestreo no probabilístico, a conveniencia y juicio del investigador (Tamayo, 2015), teniendo en cuenta las características de la población objeto de estudio, entre las cuales se enuncian los cambios de EPS por parte de los afiliados, la baja disposición de los usuarios para responder este tipo de encuestas por su estado de salud y la limitación de una base de datos para seleccionar la muestra aleatoriamente; por estos motivos, el instrumento se aplicó in situ en las diferentes EPS, tal como aparece en la tabla 3, según la disposición de los encuestados para responder.

Para recolectar la información se diseñó un instrumento estructurado tipo encuesta con 14 preguntas, con alternativas de respuesta basadas en la escala Likert, de acuerdo con la naturaleza de la variable. Este instrumento fue validado mediante juicio de expertos y, para medir su solidez interna se calculó el Coeficiente Alfa de Cronbach, con el cual se obtuvo una fiabilidad de 0,85 . A continuación se presentan, en la tabla 4 , los aspectos que se tuvieron en cuentan para operacionalizar las variables de estudio.

Tabla 4. Operacionalización de las variables de estudio

\begin{tabular}{|c|c|c|}
\hline Dimensión de análisis & Variables en las que se operacio & dimensiones de estudio \\
\hline \multirow{2}{*}{ 1. Comunicación } & Medio de comunicación con EPS & Manejo de PQR \\
\hline & Claridad de la información & Tiempo de respuesta \\
\hline \multirow{2}{*}{ 2. Tiempo (oportunidad) } & Solicitud de cita general & Urgencias \\
\hline & Solicitud con especialistas & Tiempo de atención \\
\hline \multirow{2}{*}{ 3. Profesionalismo } & Amabilidad & Interés en el paciente \\
\hline & Agilidad en la atención & Humanización del servicio \\
\hline \multirow{2}{*}{ 4. Infraestructura } & Ubicación & Higiene \\
\hline & Comodidad & Iluminación y ventilación \\
\hline \multirow{3}{*}{ 5. Eficacia } & Procedimientos & Tratamiento \\
\hline & Seguridad del paciente & Medicamentos \\
\hline & Exámenes & Precio \\
\hline
\end{tabular}

Fuente: Elaboración propia.

Como se puede observar en la tabla 4, para medir la satisfacción de los usuarios se seleccionaron 5 dimensiones: comunicación, tiempo de respuesta, profesionalismo del personal, infraestructura y eficacia, entendiéndose que esta evaluación es únicamente la de los pacientes, la cual, será necesario cotejar con los demás grupos de interés para la toma de decisiones, sin embargo, estos elementos permiten conocer la percepción de los usuarios respecto a la atención que reciben en los servicios de salud, evaluación que se concluye consultando cual es el nivel de satisfacción de los usuarios, que se mide en una escala de 1 a 5 según las siguientes opciones: 5 . Totalmente satisfecho. 4. Satisfecho 3. Indiferente 2. Insatisfecho 1. Muy insatisfecho. Esta escala se utiliza para el cálculo del índice ISAS, que se presenta en los resultados. 


\section{Análisis de resultados}

Este análisis que se presenta a continuación se basa en 5 dimensiones, comunicación, tiempo u oportunidad, profesionalismo, infraestructura y eficacia, aspectos que se estudian desde la perspectiva de los usuarios. La información se recolectó mediante la aplicación in situ, de un instrumento estructurado tipo encuesta con 14 preguntas con alternativas de respuesta basadas en la escala Likert.

Inicialmente, se consultó a los encuestados sobre la EPS a la cual están afiliados y se encontró que el 27\% están afiliados a Cafesalud-Saludcoop (actual Medimás), 23\% a Salud Total; 14\% a Nueva EPS, 11\% a Coomeva, 9\% a Sanitas, 7\% Emcosalud, 4\% a Famisanar y el 5\% restante se encuentra afiliado a regímenes especiales. En cuanto al servicio por evaluar, los resultados se distribuyeron de la siguiente forma: $33 \%$ cita médica general, 22\% urgencias, $20 \%$ especialistas, $17 \%$ odontología y $9 \%$ hospitalización, teniendo en cuenta los servicios que los usuarios habían usado recientemente.

\section{Comunicación}

Una vez abordadas las preguntas sobre la EPS y tipo de servicio, se indagó a los encuestados sobre su percepción con respecto a la gestión de las comunicaciones desde 4 aspectos: medio por el cual se comunica con la EPS, claridad de la información y gestión de peticiones, quejas y recursos - PQR y el grado de dificultad para comunicarse. Respecto al medio utilizado para contactarse con la EPS, el 54\% manifestó que se comunica por teléfono, el $32 \%$ personalmente y el $14 \%$ por Internet, resultado que permite evidenciar una tendencia positiva en la comunicación virtual con las EPS, aunque siguen predominando los medios tradicionales como las llamadas telefónicas, situación que explica la congestión y espera de los usuarios al utilizar este medio.

En cuanto a la claridad en la información, el 8.4\% de los consultados opina que la información es muy clara; el $63.8 \%$ considera que es clara; el $23 \%$ poco clara y 4,9 $\%$ opina que la información es confusa. Ese $27.9 \%$ de personas que considera que la información es poco clara y confusa, permite deducir que no se están utilizando las estrategias adecuadas para que la información sea clara, oportuna y eficaz con los usuarios de las EPS, situación que puede derivar en dificultades con los usuarios del Sistema, pues las EPS son el principal canal de comunicación con sus usuarios.

Al realizar la consulta sobre peticiones, quejas y recursos - PQR, se encontró que el 31\% había hecho algún tipo de reclamación, lo que equivale a un alto número de solicitudes. En cuanto al medio de solicitud se encontró que el $68.32 \%$ las presenta personalmente y por escrito, situación que puede congestionar la atención, si se tiene en cuenta que la mayoría de usuarios no presenta la PQR al coordinador de servicio sino al personal auxiliar que atiende asignación de citas, servicios de IPS, pagos, entre otros. En la figura 3, se puede observar los medios más usuales para la presentación de PQR. 
Figura 3. Medio usado por los usuarios para presentar $P Q R$

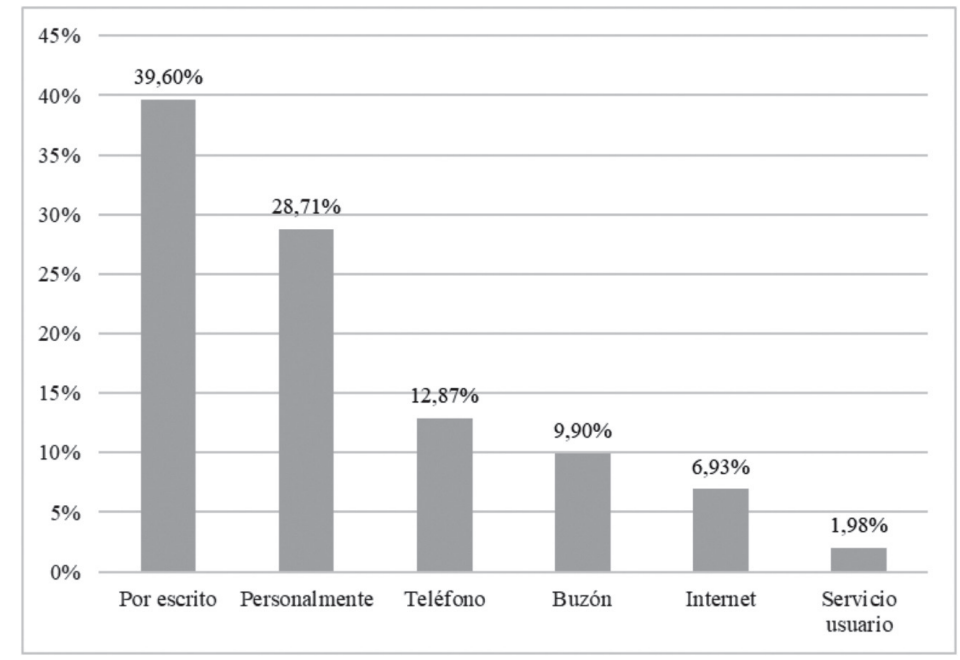

Fuente: Elaboración propia.

Otro aspecto de análisis de la variable comunicación es el grado de dificultad para presentar las reclamaciones. Respecto a esta consulta, el 31\% considera que es difícil, mientras el $69 \%$ lo considera fácil, variación que se puede relacionar con el grado de escolaridad, edad de los pacientes y el conocimiento que tienen sobre los trámites necesarios para hacer las reclamaciones. Finalmente, se consultó sobre el nivel de satisfacción que les generó la respuesta recibida por parte de la EPS al presentar una PQR y se encontró que el $28 \%$ estaba satisfecho o muy satisfecho, el $23 \%$ indiferente y el $49 \%$ insatisfecho o muy insatisfecho, como se presenta en la figura 4.

Figura 4. Nivel de satisfacción de los usuarios frente a respuestas de $P Q R$

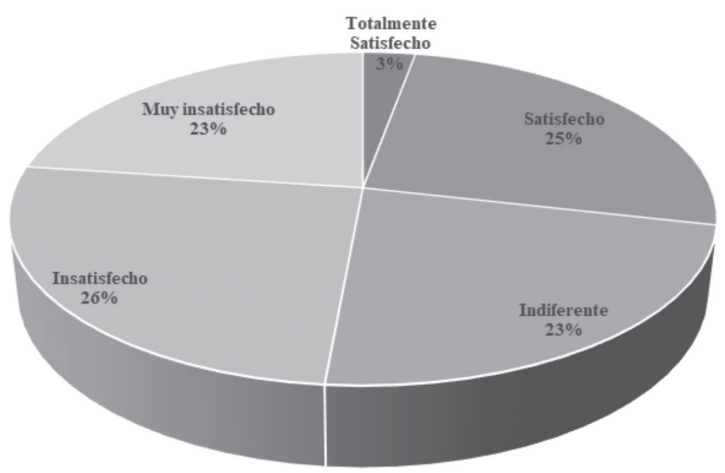

Fuente: Elaboración propia. 
Este resultado muestra un alto grado de insatisfacción por parte de los usuarios frente a los PQR, derivado de la falta de comunicación efectiva, el tiempo de respuesta, la dificultad para presentarlas y la respuesta a la reclamación. Otro indicador de este alto grado de inconformidad, es el número de usuarios que presentan derechos de petición, recursos de reposición y acuden al defensor del usuario, pero al no encontrar una respuesta satisfactoria en la EPS, acuden a entidades externas como la Defensoría del Pueblo, asociaciones de usuarios, la Procuraduría y los juzgados donde se presentan tutelas y demandas ante los jueces de la república, lo cual congestiona el Sistema Judicial, pues en 2018 se recibieron 207.734 tutelas, debido a la facilidad del trámite y celeridad de respuesta, que no puede exceder los 10 días según el artículo 86 de la Constitución Política de 1991.

\section{Oportunidad y tiempo de respuesta}

El tiempo de respuesta constituye un elemento determinante en la atención en salud, a este respecto se consultó el tiempo de asignación de citas médicas y odontológicas, encontrándose que el $55.4 \%$ espera menos de 6 días, lo que significa que al 44.6\% no se le asigna cita dentro de este plazo, incluso, el 4.4\% manifiesta que no había agenda cuando solicitó la cita, como se presenta en la tabla 5, generándose inconformidad, pues la Resolución 1552 del Ministerio de Salud y Protección Social (2013) ordena a las EPS otorgar las citas de medicina general y odontología los 3 días calendario siguientes a la solicitud, en el caso de las citas con especialistas los 5 días siguientes e informar mensualmente la oportunidad en la asignación, según las indicaciones de dicha norma.

Tabla 5. Tiempo para asignación de cita

\begin{tabular}{cc}
\hline Tiempo de espera & Porcentaje \\
\hline De 1 a 3 días & $27,8 \%$ \\
\hline De 4 a 6 días & $27,6 \%$ \\
\hline De 7 a 10 días & $23,8 \%$ \\
\hline De 11 a 14 días & $5,9 \%$ \\
\hline 15 días o más & $10,5 \%$ \\
\hline No había agenda & $4,4 \%$ \\
\hline
\end{tabular}

Fuente: Elaboración propia.

Para el caso de las solicitudes de cita con especialistas la situación no es diferente, pues cerca del $50 \%$ deben esperar más de un mes para ser atendidos, debido a que en el $17 \%$ de los casos no hay agenda o disponibilidad de especialistas, y solo el $26.2 \%$ logra obtener la cita en 15 días o menos. En la tabla 6 se presentan los resultados de esta pregunta. 
Tabla 6. Tiempo para cita con especialistas

\begin{tabular}{cc}
\hline Tiempo de espera & Porcentaje \\
\hline De 1 a 7 días & $13,2 \%$ \\
\hline De 8 a 15 días & $13,0 \%$ \\
\hline De 16 a 21 días & $12,4 \%$ \\
\hline De 22 a 30 días & $14,9 \%$ \\
\hline Más de 30 días & $29,5 \%$ \\
\hline No había agenda & $17,0 \%$ \\
\hline
\end{tabular}

Fuente: Elaboración propia.

Otro período de tiempo que se analiza es el que transcurre cuando los usuarios esperan para acceder al servicio de urgencias, sobre este se les preguntó a los encuestados: si usted o uno de sus familiares ha acudido a urgencias, ¿cuánto tiempo transcurrió antes de ser atendido? Ante esta pregunta, una tercera parte de los encuestados afirma que su espera fue mayor a 4 horas, lo cual es demasiado tiempo si es realmente una urgencia, pero en este sentido, es necesario reconocer que hay usuarios que, para ser atendidos más rápidamente acuden por urgencias sin que esté en riesgo la vida, lo cual puede colapsar el servicio de urgencias. En la tabla 7 se presenta el tiempo de espera para acceder a los servicios de urgencias.

Tabla 7. Tiempo de espera para atención en urgencias

\begin{tabular}{cc}
\hline Tiempo & Porcentaje \\
\hline Menos de una hora & $9,7 \%$ \\
\hline Entre 1 y 2 horas & $18,6 \%$ \\
\hline Entre 2 y 3 horas & $18,9 \%$ \\
\hline Entre 3 y 4 horas & $20,3 \%$ \\
\hline Más de 4 horas & $32,4 \%$ \\
\hline
\end{tabular}

Fuente: Elaboración propia.

Se observa en este caso, que más del 50\% de los pacientes deben esperar más de 3 horas para ser atendidos, aunque para solventar esta situación se atiende también mediante citas prioritarias, que representa un sobrecosto para el paciente, pero le permite ser atendido anticipadamente, cuando por su condición de salud, así lo amerite. 


\section{Profesionalismo y humanización del servicio}

Otro tema de análisis es el profesionalismo y humanización del servicio, que se refiere a aspectos como la amabilidad, respeto y celeridad que requieren los pacientes al ser atendidos; al hacer esta consulta el $67 \%$ contestó que el personal es amable, pero el $32 \%$ considera que el personal que presta servicio en las EPS no es amable con los pacientes.

Para profundizar este análisis, se consultó sobre el servicio del personal de vigilancia, información, secretarias, auxiliares y personal asistencial, encontrándose que el personal de vigilancia es el mejor evaluado, con el $78 \%$ de respuestas positivas que resultan de sumar las calificaciones buenas y excelentes, seguido del asistencial con el 74\%, mientras secretarias, auxiliares y personal de información o servicio al usuario tienen el $65 \%$ de respuestas positivas y $36 \%$ de evaluación negativa. Los resultados se presentan en la figura 5 .

Figura 5. Calificación del servicio del personal de las EPS

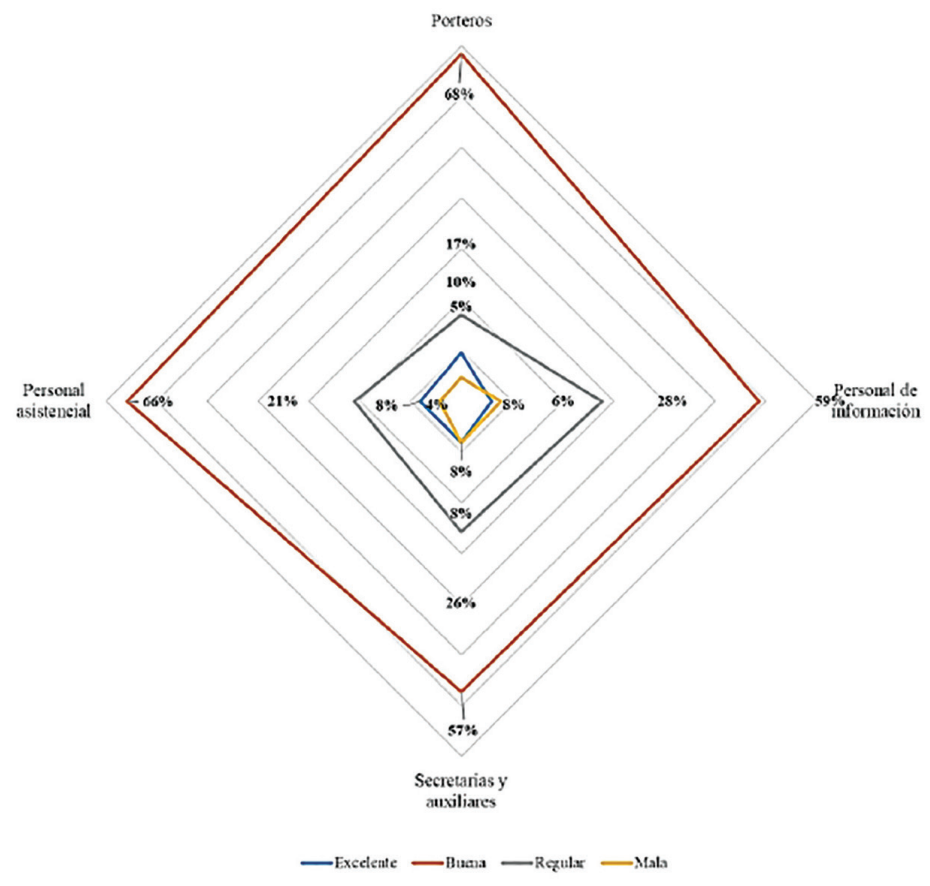

Fuente: Elaboración propia.

El promedio de estos resultados muestra que el $8 \%$ de los usuarios considera que la atención en los servicios de salud es excelente, el $63 \%$ buena, el $23 \%$ regular y el 
$6 \%$ considera que es mala, es decir que, según estos resultados, una tercera parte de los afiliados está inconforme con aspectos como amabilidad, interés en el paciente, agilidad en la atención y humanización del servicio, aspectos clave en la prestación de los servicios de salud, tal como lo reconocen Rego, Araújo y Serrão (2016).

Otro aspecto crítico en la atención en salud es el suministro de los medicamentos, sobre el cual se presentan quejas permanentes debido a la falta de disponibilidad de los mismos, la mala atención para la entrega, entre otros. Al consultar sobre este tema se encontró que el $75 \%$ considera que su atención fue oportuna y amable, pero el 17\% no lo percibió así, mientras el 8\% no respondió, debido a que no había solicitado medicamentos en el último mes. Seguidamente, se consultó respecto al profesionalismo, tiempo de dedicación, interés en el paciente, amabilidad y claridad en la información por parte del personal médico, las respuestas se presentan en la tabla 8 .

Tabla 8. Atención del médico o profesional de la salud

\begin{tabular}{ccccc}
\hline Aspecto a evaluar & Excelente & Bueno & Regular & Malo \\
\hline Profesionalismo & $13,2 \%$ & $77,0 \%$ & $8,6 \%$ & $1,2 \%$ \\
\hline Tiempo dedicado & $27,8 \%$ & $43,0 \%$ & $25,7 \%$ & $3,5 \%$ \\
\hline Interés en el paciente & $13,5 \%$ & $60,5 \%$ & $24,3 \%$ & $1,7 \%$ \\
\hline Amabilidad & $11,6 \%$ & $70,8 \%$ & $16,8 \%$ & $0,8 \%$ \\
\hline Claridad información & $11,1 \%$ & $68,4 \%$ & $18,9 \%$ & $1,6 \%$ \\
\hline
\end{tabular}

Fuente: Elaboración propia.

Se puede observar que, aunque en promedio el $63.9 \%$ de los pacientes considera que la atención en los aspectos evaluados es buena y $15.4 \%$ excelente, el $18.9 \%$ considera que es regular y $1.8 \%$ que es malo, siendo evidente que se debe mejorar. Los aspectos con mayor percepción negativa son el tiempo e interés en el paciente. Al consultar a los pacientes sobre la humanización de los servicios, se encontró que el 3\% considera que el servicio es muy humanizado, el $61.9 \%$ que es humanizado, el $26.5 \%$ que es poco humano, el $6.8 \%$ que no es humanizado y $1,9 \%$ no respondió.

Los resultados observados permiten corroborar que existen reparos por parte de un número significativo de pacientes, respecto a la calidad de la atención y humanización del servicio de salud, tal como lo reconocen Arias, Botero y Peña (2018) quienes, en su estudio sobre humanización en la prestación de servicios en salud en Pereira, Colombia, obtuvieron un promedio de $63,6 \%$ de desempeño en los aspectos evaluados. Se resalta que esta investigación se hizo en un contexto similar al del presente estudio, porque funcionan las mismas EPS, bajo la misma reglamentación y demografía poblacional símiles a las de Ibagué. 


\section{Infraestructura}

En la variable infraestructura se analizan aspectos como ubicación, comodidad, limpieza, higiene, ventilación e iluminación. Los resultados sobre esta consulta se presentan en la figura 6 , a continuación.

Figura 6. Evaluación de aspectos relacionados con infraestructura

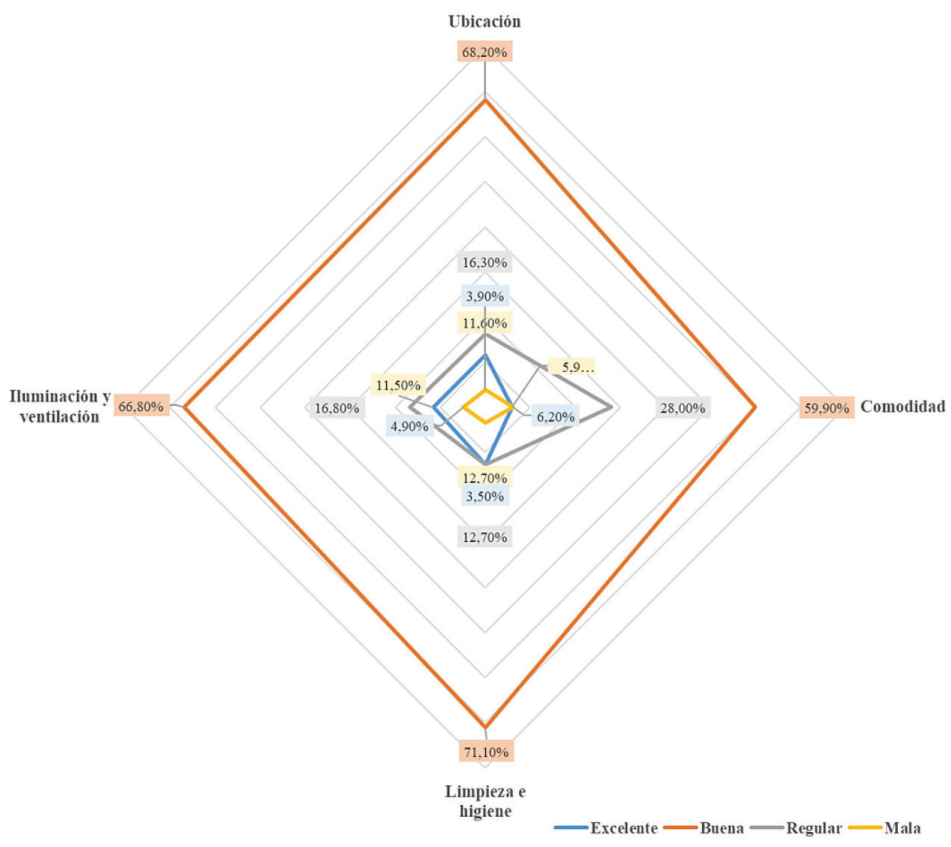

Fuente: Elaboración propia.

El aspecto mejor evaluado en este apartado es la higiene, con un $83.8 \%$ de resultados positivos y un $16.2 \%$ negativos, seguido de la ubicación, con $79.8 \%$ de respuestas positivas y $20.2 \%$ negativas. En contraste, el aspecto 'comodidad' presenta $65.8 \%$ de respuestas favorables y $34.2 \%$ desfavorables. El total de respuestas positivas con respecto a la infraestructura fue de $76.9 \%$ y negativas $23.1 \%$, resultado que revela que aspectos como ubicación, higiene, comodidad, iluminación y ventilación, son bien evaluados por gran parte de los usuarios, sin embargo, una cuarta parte de los mismos considera que hay fallas que pueden corresponder a las incomodidades que sufren muchos pacientes cuando están saturadas las oficinas, consultorios, clínicas y hospitales, incluso los servicios de urgencias donde en ocasiones los pacientes deben esperar varias horas para ser diagnosticados y atendidos. 


\section{Eficacia}

Respecto a la variable eficacia, se consultó la percepción de seguridad de los pacientes, efectividad de los procedimientos, tratamientos, medicamentos y el precio de la cuota moderadora. Los resultados obtenidos se presentan en la figura 7, a continuación.

Figura 7. Evaluación de aspectos relacionados con eficacia

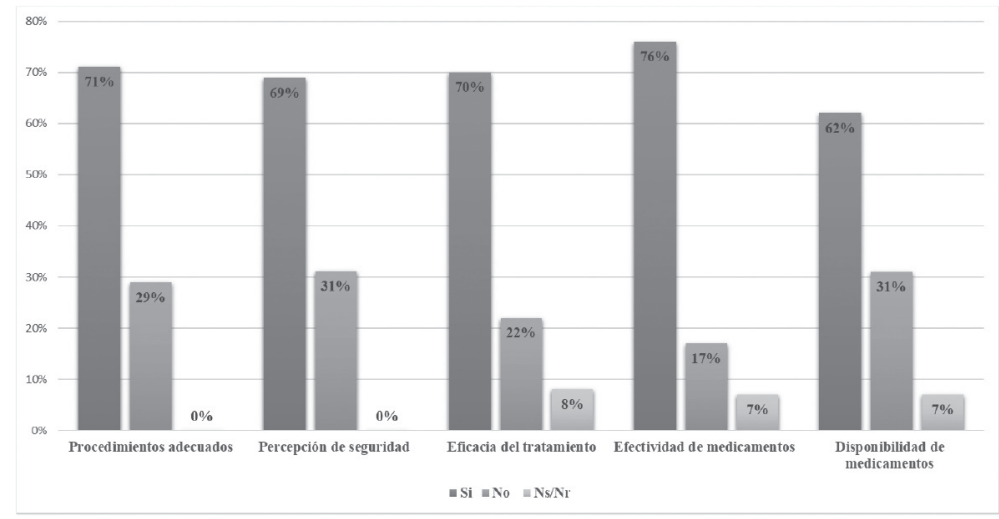

Fuente: Elaboración propia.

Se puede observar que en promedio el $70 \%$ de los afiliados evalúa positivamente los aspectos consultados con referencia a la eficacia de los servicios de salud que reciben; el mayor número de respuestas positivas se relaciona con la efectividad de los medicamentos, en contraste con el menor número de respuestas positivas que se encuentra en la disponibilidad de estos, y se resalta que el $31 \%$ de los encuestados no se siente seguro con los servicios de salud que recibe, de donde se deriva la importancia de trabajar por recuperar la confianza de los usuarios en el sistema de salud.

Con relación al precio de la cuota moderadora, que corresponde al valor que deben pagar los afiliados al utilizar los servicios de salud, según el Acuerdo 260 de 2004 del Consejo Nacional de Seguridad Social en Salud, el 8\% consideran que es muy alta, $15 \%$ la considera alta, para el $44 \%$ es moderada, el $15 \%$ la percibe como una cuota baja, 11\% manifestó que no paga esta cuota y $6 \%$ de los encuestados no respondieron esta pregunta.

Se resalta que la variación de respuestas a esta pregunta se debe a que el costo de esta cuota varía según el tipo de afiliación y base de cotización, situación que implica que quienes pertenecen al régimen subsidiado no paguen cuota moderadora, mientras quienes devenguen un salario mínimo, paguen una cuota baja y quienes devengan 2 o más salarios paguen cuotas más altas, dependiendo de sus ingresos al utilizar los servicios de salud. 
Finalmente, se preguntó a los usuarios sobre su nivel de satisfacción con el servicio de salud que reciben, entendiendo que en esta respuesta se sintetiza su percepción sobre los aspectos positivos y negativos, así como su impresión sobre la efectividad de los servicios de salud. Para cuantificar el resultado se diseñó un indicador denominado ISAS -Índice de satisfacción de atención en salud-con los resultados que presenta la tabla 9, a continuación.

Tabla 9. Nivel de satisfacción de los usuarios del servicio de salud

\begin{tabular}{ccccc}
\hline $\begin{array}{c}\text { Nivel de satisfacción de } \\
\text { los usuarios }\end{array}$ & $\begin{array}{c}\text { Número de } \\
\text { Respuestas }\end{array}$ & Porcentaje & $\begin{array}{c}\text { Indicador de } \\
\text { satisfacción }\end{array}$ & Totales por nivel \\
\hline Totalmente satisfecho & 22 & $7,5 \%$ & $100 \%$ & $7,46 \%$ \\
\hline Satisfecho & 163 & $55,3 \%$ & $75 \%$ & $41,44 \%$ \\
\hline $\begin{array}{c}\text { Indiferente } \\
\text { (ni satisfecho ni } \\
\text { insatisfecho) }\end{array}$ & $(89)$ & $\begin{array}{c}\text { Estas respuestas } \\
\text { no aporta al } \\
\text { indice en la } \\
\text { medición }\end{array}$ & & \\
\hline $\begin{array}{c}\text { Parcialmente } \\
\text { insatisfecho }\end{array}$ & 74 & $25,1 \%$ & $25 \%$ & $6,27 \%$ \\
\hline $\begin{array}{c}\text { Totalmente } \\
\text { insatisfecho }\end{array}$ & 36 & $12,2 \%$ & $0,00 \%$ \\
\hline Totales & 384 & $100 \%$ & $\begin{array}{c}\text { Índice de } \\
\text { Satisfacción } \\
\text { Salud -ISAS- }\end{array}$ & $55 \%$ \\
\hline
\end{tabular}

Fuente: Elaboración propia.

Como se puede observar, el Índice de satisfacción de atención en salud - ISAS arroja un resultado del 55\% que refleja el nivel de insatisfacción de los usuarios. Para el cálculo del ISAS se considera que, si el usuario está "Totalmente satisfecho" su aporte al indicador es 100\%, si su nivel de satisfacción es "Satisfecho", es del 75\%, la alternativa "Indiferente", se refiere a usuarios que no indicaron su nivel de satisfacción y, por lo tanto, no afectan al indicador; para este caso, como 89 encuestados se abstuvieron de responder, no se incluyeron en el ISAS. Los usuarios "Parcialmente insatisfechos" aportan el 25\% al indicador y los que están "Totalmente insatisfechos", no aportan al indicador porque su nivel de satisfacción es cero.

\section{Discusión de resultados}

La satisfacción de los usuarios en los servicios de salud es un tema de gran importancia para el Estado, es por ello que se debe estudiar desde diferentes ángulos, uno de los cuales el Gobierno, que a través del Ministerio de Salud, presentó es el Sistema 
de Evaluación y Calificación de Actores: Ranking de Satisfacción EPS 2018, según el cual los aspectos más importantes para los usuarios son oportunidad de los servicios, la facilidad y la agilidad en los trámites (Matajira, Barney, Bonett y Escobar, 2018), aspectos que también hicieron parte de esta investigación. Para la construcción del indicador, asignaron un 33.3\% a cada aspecto por evaluar: oportunidad, satisfacción y trámites. En la figura 8 se presentan los resultados del estudio citado.

Figura 8. Índice de satisfacción EPS - Estudio del Ministerio de Salud (2018)

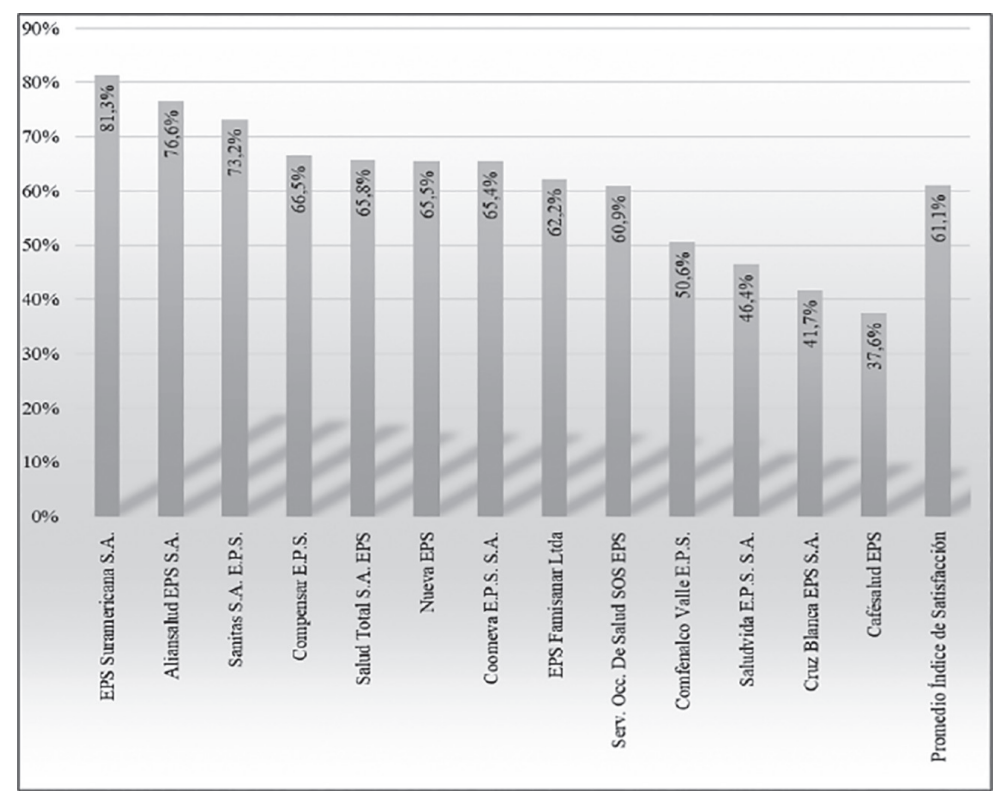

Fuente: Elaboración propia con base en Matajira, Barney, Bonett y Escobar (2018).

Como se puede observar, aunque los estudios fueron realizados de manera independiente, los resultados en los índices son símiles, lo que permite validarlos, pues mientras el promedio del Índice en este estudio fue de 55\% el del Ministerio fue de $61 \%$, lo cual corrobora que existe un alto grado de insatisfacción por parte de los usuarios de las EPS y, aunque las diferencias de los resultados no son amplias, estas se explican por los aspectos metodológicos, alcance y origen de cada investigación.

Una de las principales problemáticas del Sistema es que mientras la cobertura creció, el acceso a los servicios de salud disminuyó, pues las citas no se asignan a tiempo, los medicamentos no siempre están disponibles, al igual que el acceso a especialistas, programación de cirugías y otros aspectos que conllevan que los usuarios tengan que trasladar su PQR del Sistema de Salud al Sistema Judicial y a la Superintendencia para reclamar sus derechos, en el primer caso superando las 207.734 tutelas 
y para el segundo se calcularon 694.068 denuncias de enero a noviembre de 2019. Este panorama reafirma la necesidad de implementar medidas para que los sistemas de salud garanticen la atención efectiva, asequible y satisfactoria a los pacientes, como lo indica la OPS y lo corroboran los resultados de la investigación, que arroja un ISAS del 55\%.

Respecto a la evaluación en salud, Donabedian (1992) propuso 3 componentes los cuales fueron considerados en este estudio: la atención técnica, el manejo de la relación interpersonal y el entorno. Las principales fallas, desde la perspectiva de los usuarios, se encontraron en la comunicación, aspectos físicos, pero en gran parte, en la disposición del personal para atenderlos, lo cual contrasta con lo conceptuado por la OMS, según la cual, "se debe contar con trabajadores adecuados, competencias adecuadas, el lugar adecuado, y hacer las cosas adecuadas”, solo así, afirman Piteres, Cabarcas y Gaspar (2017), los profesionales de la salud tendrán la competencia de dar respuesta a la coyuntura actual.

Según la Defensoría del Pueblo (2018), el sistema de salud actual enfrenta grandes desafíos enfocados en mejorar el acceso y garantizar este derecho a todos los ciudadanos y permitir el más alto nivel de vida posible en salud, teniendo en cuenta las condiciones biológicas y socioeconómicas de las personas, y los recursos del Estado, entendiendo la accesibilidad como la posibilidad de acceder a los establecimientos, bienes y servicios de salud sin discriminación, desde toda zona geográfica, bajo cualquier condición económica y accediendo oportunamente a la información que requiera para su bienestar.

Finalmente, es importante considerar las posturas internacionales respecto al tema de estudio, según los cuales, mientras no garantice atención de salud de calidad y acceso real para todas las personas, no cumple con la meta de cobertura universal, según el informe de la Organización Mundial de la Salud - OMS, la Organización para la Cooperación y el Desarrollo Económicos - OCDE y el Banco Mundial - BM (2018) en el que también se afirma que las deficiencias de la atención en salud tienen un gran impacto sobre la población más pobre, observaciones que deben ser consideradas en un país como Colombia con un alto índice de desigualdad e insatisfacción en los servicios de salud, según los resultados presentados y el número de tutelas.

\section{Conclusiones}

El estudio permite concluir que el nivel de satisfacción de los usuarios es del 55\%, resultado que se explica a partir de las 5 dimensiones evaluadas desde la perspectiva de los usuarios: comunicación, oportunidad, profesionalismo, infraestructura y eficacia. Respecto a la comunicación que se encontró, que el 54\% solicita los servicios telefónicamente, lo cual congestiona la atención por este medio, cuando estas solicitudes se pueden hacer de manera virtual. También se encontró que la tercera parte de los usuarios ha presentado PQR, y de esta tercera parte, el $81.2 \%$ lo han hecho por 
escrito, personal o telefónicamente, solo el $6.9 \%$ ha presentado su PQR vía Internet, situación que congestiona aún más los servicios de salud, si se tiene en cuenta, además, que el $27.9 \%$ considera que la información que recibe es poco clara.

En cuanto a la oportunidad en la prestación del servicio se encontró que, según los usuarios, los prestadores de servicios de salud no están cumpliendo con el tiempo establecido por la ley para la asignación de citas, pues $49 \%$ manifestó que debe esperar más de 6 días para citas generales y más de un mes para el caso de citas con especialistas.

Otro aspecto que analizó es el profesionalismo y humanización del servicio, al respecto, el $32 \%$ considera que el personal no es amable. Los aspectos con más baja calificación para el personal médico están referidos al tiempo dedicado $(29.2 \%$ de calificación regular o mala), e interés en el paciente (26\% de calificación regular o mala).

Con relación a la variable profesionalismo, donde se evalúa el tiempo e interés por el paciente, la amabilidad y claridad en la información, se observa que la quinta parte de los usuarios está inconforme con este servicio y considera que se debe mejorar, siendo los aspectos con mayor percepción negativa el tiempo e interés por el paciente. Sobre la humanización, se encontró que el $26.5 \%$ considera que el servicio es poco humanizado y el $6.8 \%$ que no es humanizado, un aspecto clave en la prestación de servicios de salud, que puede influir en el estado de salud y recuperación de los pacientes.

Respecto a la infraestructura, se analizaron aspectos como ubicación, comodidad, limpieza, higiene, ventilación e iluminación, y se encontró que una cuarta parte de las respuestas son negativas, tanto en comodidad, como en ventilación, iluminación y otros aspectos que afectan la percepción de la calidad de los pacientes.

Finalmente, respecto a la eficacia en la atención que reciben los usuarios, se encontró que el $31 \%$ no se siente plenamente confiado con los servicios que recibe $\mathrm{y}$, aunque el $76 \%$ considera que los tratamiento y medicamentos son efectivos, el $31 \%$ de los encuestados manifestó que no hay disponibilidad de los medicamentos, cuando los han requerido.

A nivel general, se puede concluir que Colombia ha dado pasos importantes para garantizar la salud de la población, prueba de ello es que desde 2015 la salud es reconocida como un derecho fundamental y la cobertura alcanza estándares internacionales, pero ahora se debe avanzar hacia un servicio humanizado que genere satisfacción de los usuarios, como se indica en el ODS 3.8, sobre el derecho a tener acceso a servicios de salud de calidad; claro que la tarea no corresponde solo al Gobierno, pues como se argumentó teóricamente, el sector salud funciona como un sistema, en el cual se interrelacionan entidades públicas, privadas, empresarios, trabajadores, beneficiarios y contribuyentes, visión desde la cual todos debemos contribuir al cambio, incluso desde las universidades y la cultura ciudadana. 
Se reconoce que estos resultados muestran solo una fotografía del Sistema de Salud colombiano, desde la perspectiva de los usurarios, basados en la comunicación, oportunidad, profesionalismo, e infraestructura, para determinar un índice de satisfacción, pero este puede ser un punto de partida para generar nuevas propuestas que conlleven al mejoramiento de la atención, a partir de la generación de políticas públicas e iniciativas privadas que mejoren la calidad de los servicios de salud y el establecimiento de mecanismos para medir los progresos logrados, tal como proponen Syed, Leatherman, Mensah-Abrampah, Neilsona y Kelley (2018), según los cuales se requieren políticas dirigidas a mejorar y la coordinación de esfuerzos para un buena atención en todo el sistema.

Se concluye finalmente que el análisis del sector salud bajo el concepto de un sistema, permite identificar el origen y alcance de las fallas que han dado como resultado la insatisfacción de los usuarios, prevalencia de enfermedades, eventos adversos, el mal llamado paseo de la muerte, donde fallecen pacientes por falta de atención y quejas de los usuarios que, al no ser atendidos, acuden a los entes de control y jueces para defender sus derechos, generando traumatismo administrativos y altos costos por indemnizaciones.

\section{Referencias}

Álvarez, G. J., García, M., y Londoño, M. (2016). Crisis de la salud en Colombia: limitantes del acceso al derecho fundamental a la salud de los adultos mayores. Revista CES Derecho, 7(2), 106-125.

Arias, A., Botero, L., y Peña, L. (2018). Humanización en la prestación del servicio de salud en el área de atención prioritaria de una entidad privada de primer nivel. (Tesis de posgrado). Fundación Universitaria del Área Andina, Pereira.

Asamblea Nacional Constituyente. (1991). Constitución Política de Colombia, artículo 49. Publicada en la Gaceta Constitucional $N^{\circ} 114.4$ de julio de 1991. Bogotá: Colombia.

Ayala, J. (2014). La salud en Colombia: más cobertura, pero menos acceso. Serie Documentos de Trabajo Sobre Economía Regional, 204, 1-39. Banco de la República - Sucursal Cartagena.

Bertalanffy, V. L. (1992). Teoría general de los sistemas. (3ª reimpresión). Fondo de Cultura Económica. México.

Comité Editorial. Revista Gerencia y Políticas de Salud (2015). ¿Cuál es el propósito de nuestro sistema de salud? Revista Gerencia y Políticas de Salud. 14(28), 5-8.

Congreso de la República de Colombia (2015). Ley 1751 de 2015. Por medio de la cual se regula el derecho fundamental a la salud y se dictan otras disposiciones. Diario Oficial No. 49.427 de 16 de febrero de 2015. Bogotá: Colombia. 
Defensoría del Pueblo. (2018). La tutela y los derechos a la salud y a la seguridad social. Recuperado el 19 de diciembre de 2019, de https:/www.defensoria.gov.co/ public/pdf/Tutela-los-derechos-de-la-salud-2018.pdf

Díaz-Narváez, V. P., y Calzadilla, A. (2016). Artículos científicos, tipos de investigación y productividad científica en las ciencias de la salud. Revista Ciencias de la Salud, 14(1), 115-121. https://dx.doi.org/10.12804/revsalud14.01.2016.10

Donabedian, A. (1966). Evaluating the quality of medical care. The Milbank memorial fund quarterly, 44(3), 166-206.

Donabedian, A. (1986). Criteria and standards for quality assessment and monitoring. Quality Rev Bull (12), 99-108.

Donabedian, A. (1992). Evaluación de la calidad de la atención médica. Investigaciones sobre servicios de salud: una antología. Washington D.C.

Gayeski, M. E., Parizoto, G. M., Guimarães, G. P., Erdmann, A. L., y Meirelles, B. H. S. (2012). Sistemas de organización de cuidados en enfermería: reflexiones sobre la complejidad del cuidado como práctica asistencial. Revista Cubana de Enfermería, 28(1), 49-62.

Gómez, D. E., Pelcastre, B. E., y Parada, I. M. (2016). Diseño de una intervención gerencial para la mejora organizacional en centros de salud de primer nivel de atención. Horizonte sanitario, 15(2). 69-76.

González, V. V., Valecillos, J., y Hernández, C. (2013). Calidad en la prestación de servicios de salud: Parámetros de medición. Revista de Ciencias Sociales (Ve), 19(4), 663-671.

Kerguelén, C. A. (2008). Calidad en salud en Colombia. Los principios. Ministerio de la Protección Social. Bogotá, Colombia.

Matajira, C., Barney, E., Bonett, W., y Escobar, G. (2018). Sistema de evaluación y calificación de actores: Ranking de satisfacción EPS 2018. Oficina de Calidad, Ministerio de Salud y Protección Social. Bogotá: Colombia.

Ministerio de la Protección Social. (2004). Marco conceptual de análisis de los sistemas de salud. Proyecto de evaluación y reestructuración de los procesos, estrategias y organismos públicos y privados encargados de adelantar las funciones de vigilancia y control del Sistema de Salud. Informe final, pp. 452560. Bogotá: Colombia.

Ministerio de Salud y de la Protección Social. (2016). Resolución 429 de 2016. Política de Atención Integral en Salud. Bogotá: Colombia. Recuperado el 19 de diciembre de http://revistas.utp.edu.co/index.php/revistamedica/article/view/12711/8681

Ministerio de Salud y de la Protección Social. (2016). Resolución 1552 de 2013. Por medio de la cual se reglamentan parcialmente los artículos 123 y 124 del 
Decreto Ley 019 de 2012 y se dictan otras disposiciones. Bogotá. Recuperado el 9 de diciembre de 2019, de https:/www.minsalud.gov.co/sites/rid/Lists/ BibliotecaDigital/RIDE/DE/DIJ/resolucion-1552-de-2013.pdf

Ministerio de Salud y Protección Social. Dirección del Desarrollo del Talento Humano en Salud. (2017). Observatorio de Talento Humano en Salud OTHS Colombia. ROSS; Generalidades OTHS. Recuperado el 15 de diciembre de 2019, de https:/www.minsalud.gov.co/sites/rid/Lists/BibliotecaDigital/RIDE/VS/TH/ Guia-observatorio-talento-humano-Salud-oths.pdf

Ministerio de Salud y Protección Social. (2019). Cifras de aseguramiento en salud. Bogotá Colombia. Recuperado el 12 de noviembre de 2019, de https://www. minsalud.gov.co/proteccionsocial/Paginas/cifras-aseguramiento-salud.aspx

Morales, L. G., y García, J. C. (2017). Gestión de la crisis del sistema de salud en Bogotá. Revista de Salud Pública, 19(1), 129-133. https://doi.org/10.15446/rsap. v19n1.64102

Moreno, G. A. (2016). El nuevo modelo de Atención Integral en Salud - MIAS para Colombia ¿La solución a los problemas del sistema? Revista Médica de Risaralda, 22(2), 73-74.

Organización de las Naciones Unidas - ONU. (2015). Transformar nuestro mundo: la Agenda 2030 para el Desarrollo Sostenible. Resolución 70/1 aprobada por la Asamblea General el 25 de septiembre de 2015. Recuperado el 19 de diciembre de 2019 de https://undocs.org/sp/A/RES/70/1

Organización Mundial de la Salud - OMS, Organización para la Cooperación y el Desarrollo Económicos - OCDE y Banco Mundial - BM. (2018). Delivering quality health services: a global imperative for universal health coverage. Ginebra: Suiza. Recuperado el 20 de octubre de 2019, de https:/apps.who.int/iris/ bitstream/handle/10665/272465/9789241513906-eng.pdf?ua=1\&ua=1

Organización Mundial de la Salud - OMS, Organización Panamericana de la Salud OPS. (2018). Evidencia e inteligencia para la acción en salud. Análisis de salud, métricas y evidencia: situación de salud en las Américas: Indicadores básicos 2018. Washington: Estados Unidos.

Palmer, R.H. (1989). Evaluación de la asistencia ambulatorio. Principios y práctica. Ministerio de Sanidad y Consumo. Ministerio de Sanidad y Consumo. Madrid, España.

Piteres, R., Cabarcas, M., y Gaspar, H. (2017). El recurso humano factor de competitividad en el sector salud. Investigación e Innovación en Ingeniería, 6(1). 93-101. https://doi.org/10.17081/invinno.6.1.2778.

Prada, S. I., Salinas, M. A. (2016). Estadísticas del sistema de salud: Colombia frente a OCDE. Documentos de trabajo Proesa (10), 1-26. 
Pretelt, J. (2017). El derecho a la salud en Colombia: evolución y defensa frente a un estado que dista de ser garante. Revista Avances en Salud, 1(2), 51-54. https://doi. org/10.21897/25394622.1222

Ramos, B. N. (2011). Calidad en la atención médica. Control de la calidad de la atención en salud. (2 ${ }^{\mathrm{a}}$. ed.). La Habana, Cuba: Editorial Ciencias Médicas.

Rego, A., Araújo, B., y Serrão, D. (2016). The mission, vision and values in hospital management. Journal of Hospital Administration, 5(1), 62-72. https://doi. org/10.5430/jha.v5n1p62

Suárez, L. F., Puerto, S., Rodríguez, L. M., y Ramírez, J. (2017). La crisis del sistema de salud colombiano: una aproximación desde la legitimidad y la regulación. Revista Gerencia y Políticas de Salud, 16(32), 34-50. http://dx.doi.org/10.11144/ javeriana.rgps16-32.cssc

Superintendencia de Salud (2019). Comportamiento de peticiones, quejas, reclamos o denuncias (PQRD) y solicitudes de información formuladas por los usuarios del Sistema Nacional de Salud ante la Supersalud en el período comprendido entre enero y noviembre de 2019. Bogotá, Colombia. Recuperado el 19 de diciembre de 2019, de https://www.supersalud.gov.co/es-co/nuestra-entidad/ cifras-y-estadisticas

Syed, S., Leatherman, S., Mensah-Abrampah, N., Neilson, M., y Kelley, E. (2018). Improving the quality of health care across the health system. Bull World Health Organ, 96(799). http://dx.doi.org/10.2471/BLT.18.226266

Tamayo, G. (2015). Diseños muestrales en la investigación. Semestre Económico $4(7), 1-14$.

Triviño, A. F. R. (2015). Coyuntura del sistema de salud en Colombia: caracterización de una crisis desde las particularidades financieras de las EPS. Gestión \& Desarrollo, 10(1), 103-116.

Yepes, C. E., y Marín, Y. A. (2018). Desafíos del análisis de la situación de salud en Colombia. Biomédica, 38(2), 162-172. 